\title{
Pulse Transit Time (PTT) Measurements During Laparoscopic and Open Abdominal Surgery: A Pilot Study in ASA I-II Female Patients
}

\author{
Marnix Sigtermans, Jasper Looijestijn, Erik Olofsen and Albert Dahan* \\ Department of Anesthesiology, Leiden University Medical Center, 2300 RC Leiden, The Netherlands
}

\begin{abstract}
Background: The pulse transit time (PTT) is the time interval between the R-wave of the ECG and the appearance of the pulse wave in the periphery during the same cardiac cycle. PTT is influenced by various factors including sympathetic tone and vascular compliance. The authors assessed the effect of anesthesia, intubation and laparoscopic (LPS) versus laparotomic (LPT) surgery on PTT.

Methods: PTT, mean arterial blood pressure (MAP) and heart rate (HR) were measured during induction of anesthesia and during the first 45-min of LPS $(n=17)$ and LPT $(n=13)$ surgery in a group of female patients.

Results: Anesthesia increased PTT values, while painful stimuli caused an immediate reduction. During surgery, we observed group effects on PTT and MAP with $10-25 \%$ lower PTT values $(P<0.001)$ and $15-30 \%$ greater MAP values $(P<$ $0.01)$ in the LPS group. HR did not differ between groups.

Conclusions: The pulse transit time was used successfully to track the effect of anesthesia and stressful nociceptive stimuli during induction of anesthesia. The shorter PTT values during laparoscopic abdominal surgery compared to open abdominal surgery suggest a higher sympathetic tone in patients undergoing laparoscopic surgery and/or an effect of the increased abdominal pressure on PTT.
\end{abstract}

Keywords: Pulse transit time, laparoscopy, open abdominal surgery, sympathetic tone, intraabdominal pressure.

\section{INTRODUCTION}

The pulse transit time (PTT) is the time interval between the R-wave of the ECG and the appearance of the pulse wave in the periphery (for example, the finger tip) during the same cardiac cycle. PTT was first introduced in the 1950s in psycho-physiological studies on anxiety and stress $[1,2]$. Since the 1990s it is used to measure sympathetic activation during upper airway obstruction in sleep [3]. The use of pulse transit time monitoring during general or local anesthesia is relatively new [4-7]. The results of studies performed during general anesthesia indicate that PTT changes with anesthetic depth $[4,6]$, while studies performed during general and spinal anesthesia suggest that PTT reflects autonomic tone and may function as a surrogate marker of arterial blood pressure $[5,6]$. Furthermore, Singham and coworkers showed that PTT responds to nociceptive stimulation independently of heart rate [5]. Although there seems general consensus in the notion that PTT reflects sympathetic tone [1-7], using PTT as a marker for blood pressure is still open to debate with some authors arguing that the PTT is just too unreliable or inaccurate to function as an index of blood pressure $[8,9]$.

To get a further indication of the effect of anesthesia and surgery on PTT we measured PTT values during general anesthesia for open and laparoscopic abdominal surgery. We initially measured changes in PTT that occurred due to induction of anesthesia and laryngoscopy/ intubation. Next we

*Address correspondence to this author at the Department of Anesthesiology, Leiden University Medical Center, P5-Q, 2300 RC Leiden, The Netherlands; Fax: +31 7152624 48; E-mail: a.dahan@lumc.nl compared PTT values during surgery in the laparoscopic (LPS) and laparotomic (LPT) groups. We choose to compare LPS and LPT abdominal surgeries for the reason that there are data that show the presence of differential stressful stimuli between the two procedures $[10,11]$. For example, during the first postoperative hours laparoscopic surgery is associated with more severe pain and increased analgesic requirements compared to patients after a laparotomy [10]; and during surgery LPS abdominal surgery is associated with enhanced metabolic and stress hormonal responses compared to open surgery [11]. In the current study we use the pulse transit time as a marker of sympathetic tone and a possible surrogate marker of the nociceptive state of the patient and consequently expect significant differences in PTT values between the two surgical techniques.

\section{METHODS}

\section{Patients}

The protocol was approved by the local Human Ethics Committee. Since the measurements that we collected were part of the normal standard care and the study was purely observational no informed consent was deemed necessary. Thirty female patients, ASA I or II, scheduled for elective abdominal surgery were included in the study. The sample size was calculated using SamplePower 2.0 (SPSS Inc.) assuming a $30 \%$ difference between surgical techniques in PTT value, SD of $20 \%$, alpha $=0.05$ and beta $>0.80$. Inclusion criteria were: patient $18-65$ years, body mass index < 30 , absence of cardiovascular disease; exclusion criteria were: the use of illicit drugs, the use of medication that could influence our measurements (e.g., $\beta$-blockers, atropine). 


\section{Anesthesia and Surgery}

None of the patients received premedication. General anesthesia was induced according to the following guideline: remifentanil at $10 \mu \mathrm{g} \cdot \mathrm{kg}^{-1} \cdot \mathrm{h}^{-1}$, followed by an induction dose of propofol $(2 \mathrm{mg} / \mathrm{kg})$ and atracurium $(0.5 \mathrm{mg} / \mathrm{kg})$. The trachea of all patients was subsequently intubated (tube sizes 89) and propofol was continued at an infusion rate of $6-10$ $\mu \mathrm{g} . \mathrm{kg}^{-1} \cdot \mathrm{h}^{-1}$. Since this was an observational study, the attending anesthesiologist was allowed to change the drug doses and infusion rates according to his own discretion. His or her decisions were based on the routine parameters used to guide anesthesia (heart rate, blood pressure, patient movement, sudomotor responses). Furthermore, since the patients were not randomized (the selection of the operational technique was made by the surgeon in a phase prior to patient selection) we consider our study a pilot study.

The patients underwent either laparoscopy surgery (LPS, $n=17$ ) or an elective laparotomy (LPT, $n=13$ ) for various procedures. In the laparoscopic group, insufflation of carbon dioxide into the abdominal space was such that abdominal pressure remained between 12 and $16 \mathrm{mmHg}$. No wound infiltration with local anesthetics was used prior to incision.

\section{Monitoring and Data Acquisition}

Continuous 3-lead ECG and infrared pulse oximeter waveforms (obtain from the index finger of the left hand) were obtained from a Cardiocap II and a Capnomac Ultima device (Datex, Helsinki, Finland). These signals were linked to a custom made analogue computer (Marc Geerts, LUMC, The Netherlands), which calculated the pulse transit time for each heart beat. The PTT was calculated as the time between the R-wave of the ECG (lead II) and the upstrokes of the waveform of the pulse oximeter of the same cardiac cycle (the upstroke was determined and the time at which $50 \%$ of the upstroke occurred was set at the cut-off value for PTT measurement, see also [3]). The algorithm was validated on simulated data. PTT values and R-R interval were collected on the hard disc of a computer for further analysis. An algorithm in the PTT calculation program allowed for the automatic removal of artifacts related to abnormal ECG and pulse oximeter signals. The artifacts were related to movement of the hand or electrocoagulation causing disturbances in the pulse oximeter and ECG signals (about $5 \%$ of the data was rejected due to the occurrence of artifacts related to the absence of a usable R-wave of the ECG or an irregular upstroke of the plethysmogram). Other measurements that were collected for data analysis were blood pressure (Datex Cardiocap II), end-tidal carbon dioxide concentration (Datex Capnomac Ultima), and oxygen saturation (Capnomac U1tima). These variables were collected at 5 min intervals.

Data collection started 5 to 10 -min prior to the induction of anesthesia and continued for 45 minutes from the start of the surgical incision. The data obtained at seven time points were used in the statistical analysis: 1. baseline (prior to any drugs give); 2. just prior to intubation (induction); 3. laryngoscopy and intubation; 4. just prior to incision (deepening of anesthesia); 5. $15 \mathrm{~min}$ into surgery; 6. $30 \mathrm{~min}$ into surgery; and 7. $45 \mathrm{~min}$ into surgery. We restricted the study to the first 45-min of surgery since half of the laparoscopic procedures just didn't last longer than $50 \mathrm{~min}$.

\section{Data Analysis}

PTT values at the seven time points were averaged for 10 s. These values were used in the statistical analysis. The data obtained around intubation and during surgery were analyzed separately (intubation data points $1-4$; surgery data points $4-7$ ). To detect a significant difference between treatments (LPS versus LPT) and among the different data points we performed a two-way analysis of variance with post hoc t-tests with Bonferonni corrections (Sigmastat 3.1, Systat Software, Inc., San Jose, CA, USA). The Pearson correlation coefficient was calculated to obtain information on the correlation between blood pressure and PTT during the second phase (surgery) of the study. Receiver operating characteristic (ROC) curves were calculated for the change in PTT in relation to an increase in MAP by 5 and $10 \%$ using the MedCalc statistical software package (MedCalc Software, Mariakerke, Belgium). $P$-values $<0.05$ were considered significant. Values are mean \pm standard deviation (SD) unless otherwise stated.

\section{RESULTS}

The two study groups did not differ with respect to patient age, BMI, ASA class and the amount of remifentanil and propofol used during anesthesia induction and the first 45 min of surgery (see Table 1). The type of surgical procedures did differ between the two groups (Table $\mathbf{1}$ ).

\section{Induction of Anesthesia and Insertion of the Endolaryn- geal Tube}

In Fig. (1) the effect of induction of anesthesia and surgery on cardiovascular measurements in a patient that underwent an open procedure for a hysterectomy is shown. The data obtained during induction (data points 1 to 4 ) are typical for the whole study group. Anesthesia caused the increase in PTT values (data points 2 and 4), while insertion of the endolaryngeal tube and gastric tube caused an immediate decrease in PTT (data point 3). During this phase of the study, heart changes were in accordance (but reciprocal) to the changes in PTT while blood pressure values (obtained at 5-min intervals) showed a gradual decrease. The mean values of the study population are given in Fig. (2). Insertion of the endolaryngeal tube was associated with a significant decrease in pulse transit time from $268 \pm 33$ to $235 \pm 30 \mathrm{~ms}(P$ $=0.013)$ and an increase in heart rate from $72 \pm 13$ to $88 \pm$ 19 beats $/ \mathrm{min}(P=0.002)$ while no change was observed in blood pressure.

\section{Laparoscopic vs Open Procedures}

The mean values of the measured variables obtained at the four data points during laparoscopic and laparotomic surgery are shown in Fig. (3). We present the values of the pulse transit time relative to baseline to take into account the variability that occurred among subjects in baseline PTT values (that is, PTT at the end of induction, data point 4). As expected end-tidal $\mathrm{PCO}_{2}$ values were about $0.5 \mathrm{kPa}$ greater in the laparoscopic group $(P<0.01$ versus LPT). Group effects were further present on PTT and blood pressure with $10-25 \%$ lower PTT values in the laparoscopic group $(P<$ $0.001)$ and $15-30 \%$ greater mean arterial blood pressure values in the patients undergoing a laparoscopic procedure $(P<$ $0.01)$ compared to patients undergoing an open procedure. 
Table 1. Characteristics of the Laparoscopic and Laparotomic Surgery Study Groups

\begin{tabular}{|c|c|c|c|}
\hline & Laparoscopy & Laparotomy & $P$-Value \\
\hline Number of patients & 17 & 13 & ns \\
\hline Age (SD) & $49(16)$ & $44(10)$ & ns \\
\hline $\mathrm{BMI}(\mathrm{SD})$ & $27(5)$ & $25(4)$ & $\mathrm{ns}$ \\
\hline ASA I/II & $12 / 5$ & $10 / 3$ & \\
\hline \multirow[t]{4}{*}{ Procedures $(n)$} & cholecystectomy (3) & hysterectomy (5) & \\
\hline & nephrectomy (2) & urethrotomy (1) & \\
\hline & adnex surgery (9) & exploratory surgery (5) & \\
\hline & colonic surgery (6) & cholecystectomy (2) & \\
\hline Propofol dose (SD) $\mathrm{mg} / \mathrm{kg}$ * & $9.8(2.4)$ & $9.8(3.8)$ & ns \\
\hline Remifentanil dose (SD) $\mathrm{mg} / \mathrm{kg}$ * & $14.3(2.7)$ & $16.2(6.9)$ & ns \\
\hline Atracurium dose (SD) $\mathrm{mg}$ * & $45(5)$ & $43(8)$ & ns \\
\hline BIS values (range) & $44-57$ & $39-60$ & ns \\
\hline Duration of surgery (range) $\mathrm{min}$ & $78(50-135)$ & $124(55-180)$ & ns \\
\hline
\end{tabular}

* Total dose given from induction until the end of the study (45 min into surgery).

No effect of the surgical procedures was observed on heart rate.

\section{Pulse Transit Time versus Blood Pressure}

The relationship between PTT and mean arterial pressure is given in Fig. (4). There was significant albeit small correlation with PTT with $\mathrm{R}^{2}=0.30(\mathrm{df}=76, P<0.001)$.
We constructed receiver operating curves (ROC) to get an indication of the sensitivity and specificity of PTT to reflect $5 \%$ and $10 \%$ changes in mean arterial blood pressure (Fig. 5). For an increase in MAP of $10 \%$, a change in PTT of $5 \%$ had a sensitivity of $91 \%$ and specificity of $62 \%$, while a change in PTT of $10 \%$ had a sensitivity of $77 \%$ and specificity of $71 \%$. The corresponding values for an increase in MAP of $5 \%$ were $93 \%$ and $68 \%$ (sensitivity and specificity

\section{Abdominal surgery - non laparoscopic}
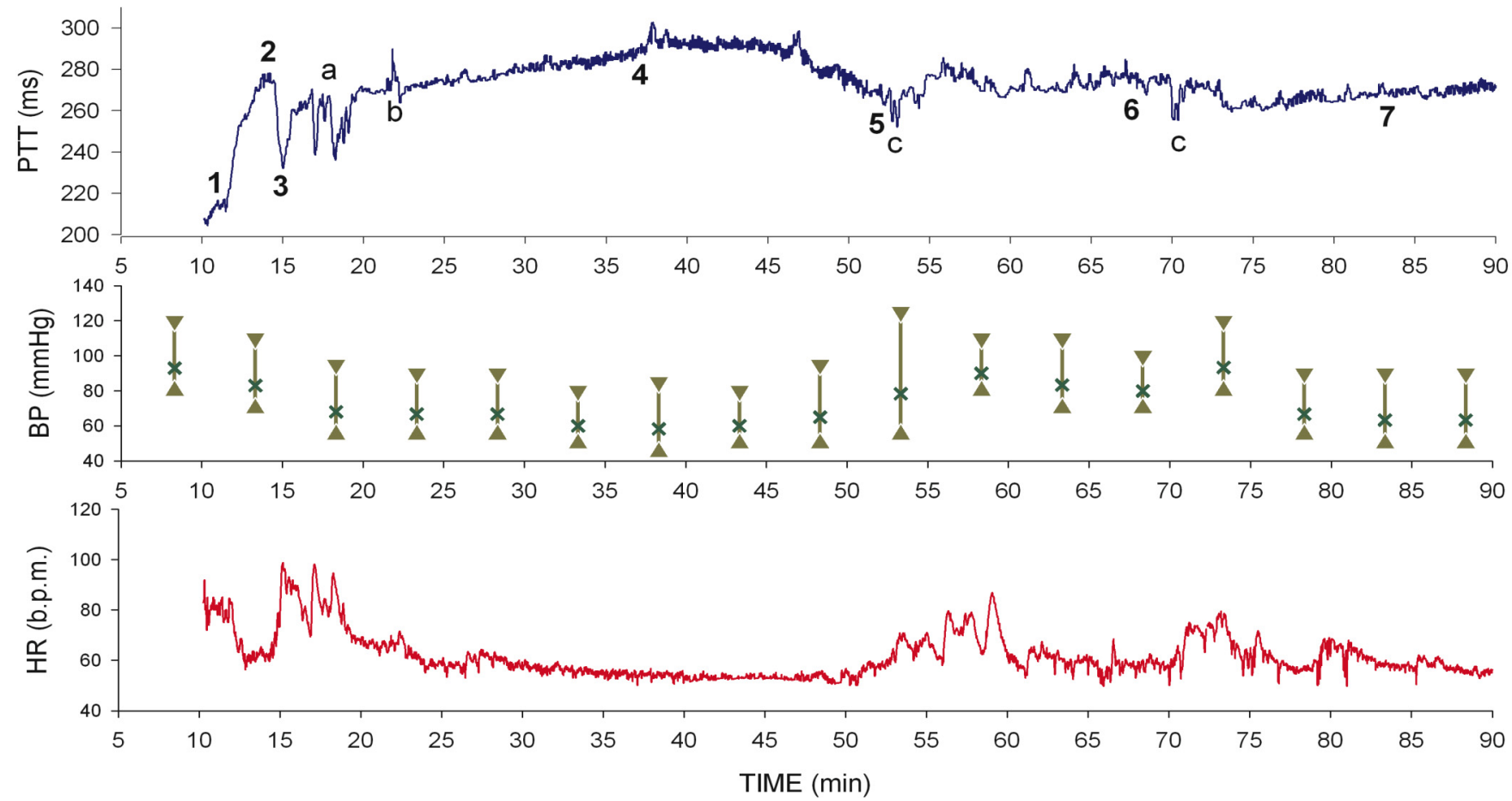

Fig. (1). Example of the pulse transit time (PTT) values during induction of anesthesia and the first 45-min of surgery in an non-laparoscopic abdominal procedure. Also given are the blood pressure values $(X$ is mean arterial pressure) and heart rate values (1/R-R interval as obtained from the PTT computer). 1 = predrug baseline, $2=$ induction of anesthesia just prior to intubation, $3=$ laryngoscopy and intubation; $4=$ just prior to incision, $5=15 \mathrm{~min}$ into surgery, $6=30 \mathrm{~min}$ into surgery and $7=45 \mathrm{~min}$ into surgery. a $=$ multiple attempt to introduce a gastric tube, $\mathrm{b}=$ artifact, $\mathrm{c}=$ peritoneal traction. 
Pulse Transit Time

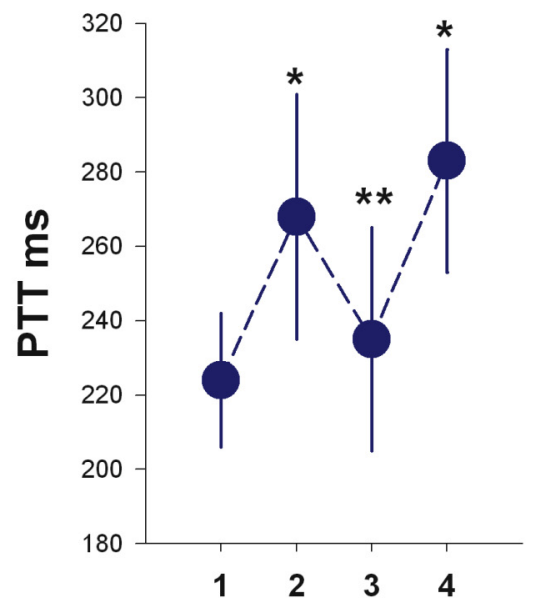

Mean Arterial Pressure

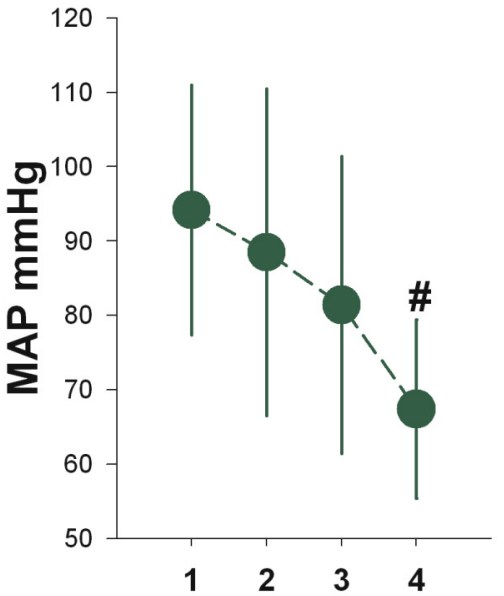

Heart Rate

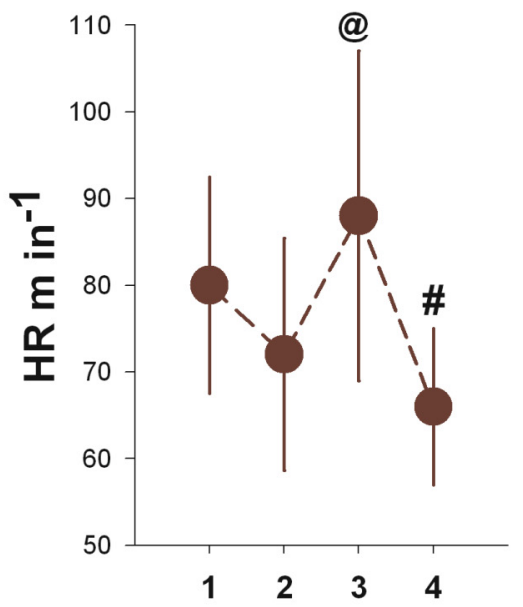

Fig. (2). The effect of anesthesia induction, laryngoscopy/intubation and deepening of anesthesia on pulse transit time (PTT, left), mean arterial pressure (MAP, middle) and heart rate (HR, right). $\mathbf{1}=$ predrug baseline, $\mathbf{2}=$ induction of anesthesia just prior to intubation, $\mathbf{3}=$ laryngoscopy and intubation; 4 = just prior to incision. PTT: ${ }^{*} P<0.01$ versus $1,{ }^{* *} P<0.02$ versus 2 and 4 ; MAP: \# $P<0.01$ versus 1 ; HR: \# $P<$ 0.01 versus $1, @ P<0.01$ versus 2 and 4.
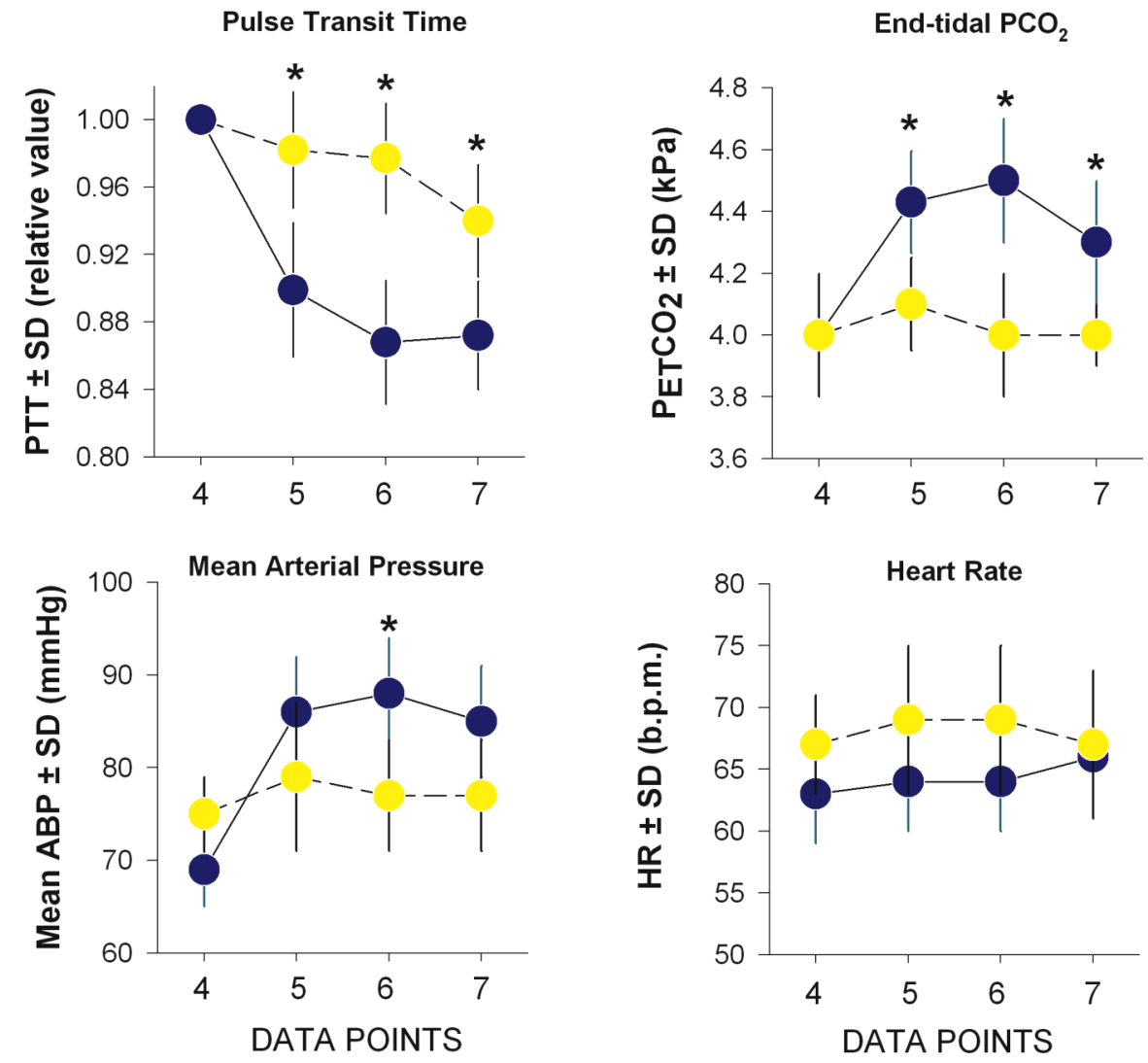

Fig. (3). Pulse transit time (PTT), end-tidal $\mathrm{PCO}_{2}$, blood pressure and heart rate (HR) during laparoscopic surgery (blue) and laparotomic (yellow) surgery. $\mathbf{4}=$ just prior to incision, $\mathbf{5}=15 \mathrm{~min}$ into surgery, $\mathbf{6}=30 \mathrm{~min}$ into surgery, $7=45$ min into surgery and end of study. The comparisons shown are between treatment levels (i.e., laparoscopic versus non-laparoscopic) of the same time period. ${ }^{*} P<0.01$. Between group comparisons revealed a significant treatment effect on PTT (treatment and treatment*time: $P<0.001$ ), blood pressure (treatment*time: $P<0.01$ ) and end-tidal $\mathrm{PCO}_{2}$ (treatment and treatment*time: $P<0.05$ and $P<0.01$, respectively).

of a $5 \%$ change in PTT) and $82 \%$ and $78 \%$ (sensitivity and specificity of a $10 \%$ change in PTT). For the $5 \%$ MAP change, the area under-the-curve is 0.85 (95\% confidence interval $=0.75-0.92)$; for the MAP $10 \%$ change the corresponding values are $0.79(0.68-0.87)$. Both areas are significantly greater than $0.5(P<0.0001)$. 


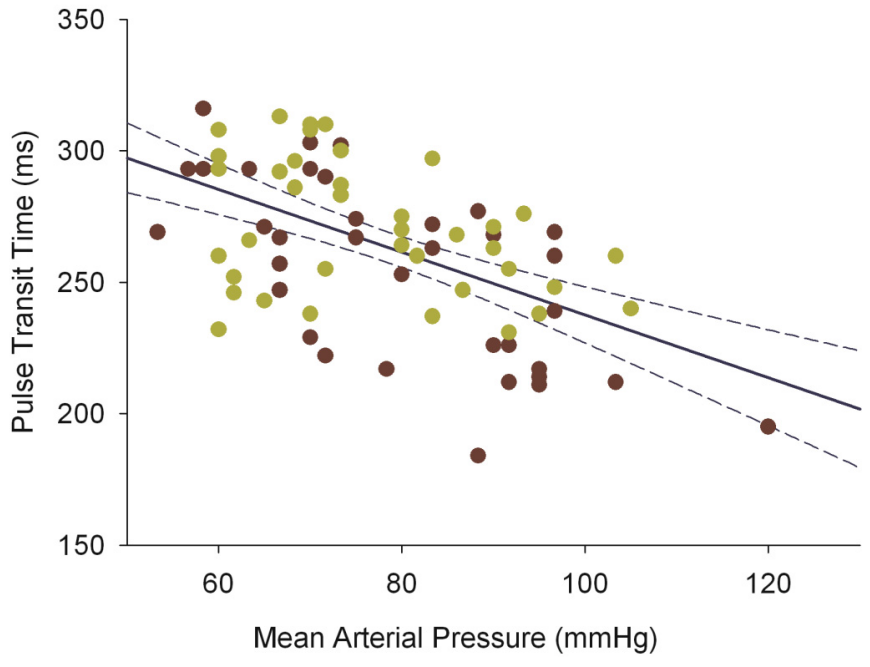

Fig. (4). Blood pressure versus pulse transit time during the $2^{\text {nd }}$ part of the study (surgery). Dark red symbols: Laparotomies. Dark yellow symbols: Laparoscopies. Continuous lines are the linear regressions, broken lines, the $95 \%$ confidence intervals. The $\mathrm{R}^{2}$ value is $0.30(\mathrm{df}=76, \mathrm{P}<0.001)$.

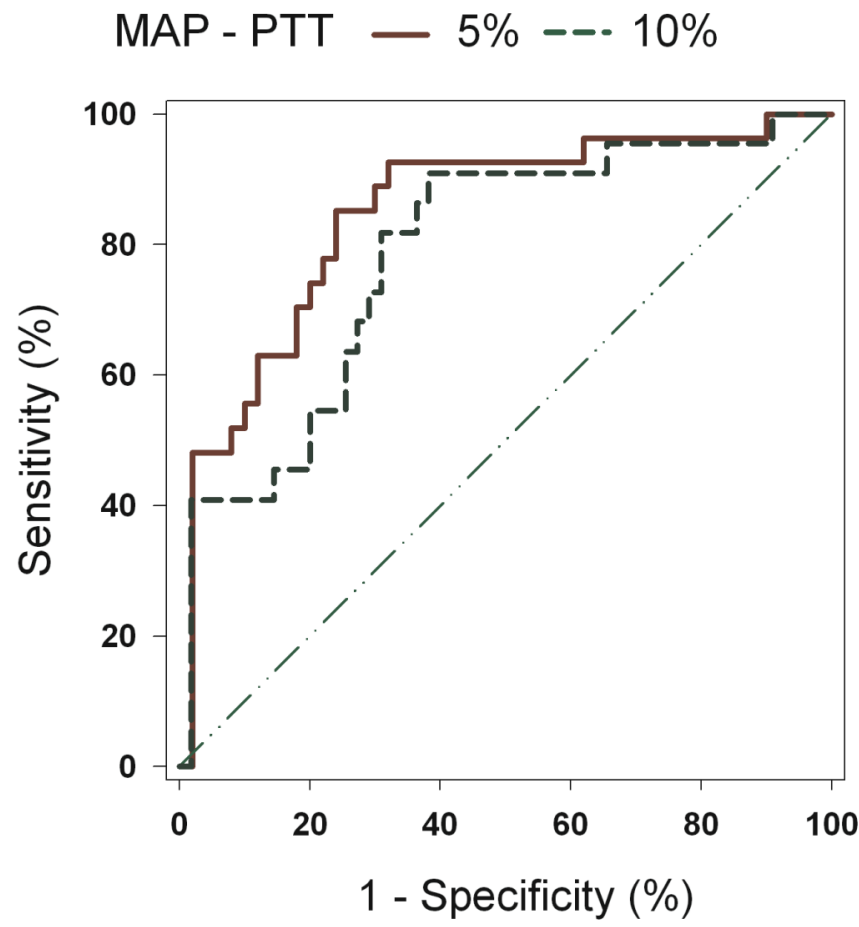

Fig. (5). A. Receiver operating characteristics (ROC) for the change in pulse transit time in relation to an increase in blood pressure by 5 (continuous dark red line) and 10\% (broken dark green line). Data are from the surgical phase of the study. For the 5\% change, the area under-the-curve is 0.85 (95\% confidence interval $=0.75-$ $0.92)$; for the $10 \%$ change the corresponding values are $0.79(0.68-$ $0.87)$. Both areas are significantly greater than $0.5(P<0.0001)$.

\section{DISCUSSION}

In the current study we measured the pulse transit time during induction of anesthesia, laryngoscopy/intubation and during laparoscopic and laparotomic surgery. We observed in the first part of the study that anesthesia lengthens PTT while in- sertion of the endolaryngeal tube (and other painful stimuli) caused its shortening. In the second part of the study we observed a significant difference between PTT values in the LPS group compared to the LPT group.

We used a custom made analogue PTT computer which calculated the PTT by taking the time interval between the Rwave of the ECG and the appearance of the pulse wave in the finger tip. By using the R-wave in the calculation of the PTT, the PTT includes the electrical activation and isovolumetric contraction of the ventricle (pre-ejection phase, PEP), mechanical ventricular ejection (ejection phase), the propagation of the pulse pressure wave to the periphery (propagation or vascular phase) and the upstroke of the plethysmogram (in our algorithm the time that the upsweep was at $50 \%$ of its maximum was chosen as cut-off value) [7,8]. The pre-ejection period (PEP) and ejection phase (i.e., the non vascular components of PTT) take up about 10 to $35 \%$ of the PTT. The PTT is determined by many factors such as preload, afterload, heart rate and cardiac contractility (these influence the PEP and ejection phases of the PTT), blood density and vascular elastance (these influence the propagation phase of PTT) [7,8]. Vascular elastance is dependent on compliance of the vessel, arterial blood pressure and sympathetic tone. In contrast with older studies on PTT, we used a PTT computer that incorporated an artifact rejection algorithm. This increased the reliability of the PTT calculations. Our device is identical to that used by Singham and coworkers and Sharwood-Smith and coworkers $[5,6]$. However, a custom made PTT computer is not needed for its measurements as it may easily be trended from suitable designed patient monitors.

Our observation that PTT increases with anesthesia (Figs. 1 and 2 ) is in agreement with earlier findings during general and spinal anesthesia $[5,6]$. It indicates that PTT is sensitive to sympathicolysis from anesthesia. Furthermore we observed rapid shortening of PTT in response to nociceptive stimulation (insertion of laryngoscope, intubation, insertion of naso-gastric tube, Figs. (1 and 2)). Our current finding concur with a short report where we showed that PTT correlates well with bispectral index (BIS) values [4]. However, in contrast to BIS, low PTT values correlate well with perceived nociceptive stimulation such as intubation and patient movement. This was confirmed by Singham and coworkers, who argued that PTT reflects autonomic or sympathetic responses to nociceptive stimulation [5].

Sharwood-Smith and coworkers [6] showed that the PTT gives a reliable beat-to-beat indication of arterial blood pressure during spinal anesthesia for Cesarean Section. They further showed that PTT allowed an accurate predication of the onset of hypotension. In our study PTT did not reflect arterial blood pressure as well as in the study of Sharwood-Smith and coworkers: PTT was unable to account for $70 \%$ of blood pressure variance (Fig. 4) with specificity and sensitivity values ranging around $70 \%$ (Fig. 5). A recent study in healthy volunteers showed that a major draw back of the use of PTT might be that its correlation with mean arterial pressure becomes unreliable during infusion of vasoactive drugs (such as noradrenalin and salbutamol) [8]. Our observations and those of others $[8,9]$ indicate that using of PTT as a surrogate measure arterial blood pressure may not be reliable, at least not under all circumstances. 
A cause for the shorter PTT values in the LPS group may be related to a higher sympathetic tone during LPS compared to LPT. An increase in sympathetic tone may occur due to the sustained pressure from the pneumo-peritoneum on retroperitoneal and para-aortal nerve tissue, splanchnic bed, abdominal and retroperitoneal viscera [10]. Similarly, the $\mathrm{CO}_{2}$ absorbed from the peritoneal cavity may cause sympathico-excitation [12]. We also need to consider other causes for the shortening of PTT unrelated to a direct effect of sympathetic tone on PTT. The increased abdominal pressure may have affected PTT either via changes in preload (causing a shortening of PEP) or due to the requirement for higher intrathoracic pressures for a given tidal volume [13-15]. A higher intrathoracic pressure will affect PTT by changing PEP as well as by changing the compliance of the arterial blood vessels [13-15]. Our study design does not allow us to differentiate between PTT changes from an increased sympathetic tone or from an increased abdominal and thoracic pressure. We therefore argue that the differences in PTT between LPS and LPT group were caused by the effect of the increased abdominal pressure combined with an effect of an increased sympathetic tone.

\section{Critique of Methods}

We performed our studies exclusively in female patients. This was done for practical reasons (we aimed at predominant gynecological laparoscopic procedures) and to exclude possible gender effects of the analgesic regimen [16]. We restricted our studies to the first $45 \mathrm{~min}$ of surgery. As stated earlier this was done for the simple fact that most of the laparoscopic procedures last no longer than $50 \mathrm{~min}$. In those cases in which the laparoscopic procedure lasted longer than $50 \mathrm{~min}$ PTT values did remain within the range observed during the initial part of surgery (laparoscopy PPT range from 0.78 to 0.90 of control versus laparotomy PTT 0.92 to 1.2 of control). Finally, it may be argued that patients in the laparoscopy groups received inadequate anesthesia and consequently had lower PTT values. We do not believe that this was the case. Bispectral index values were routinely monitored in our patients and the BIS values did not differ between groups (see Table 1). This indicates that the depth of anesthesia was similar between the two groups.

\section{CONCLUSIONS}

The pulse transit time was used successfully to track the effect of anesthesia and stressful nociceptive stimuli during induction of anesthesia. During laparoscopic abdominal surgery PTT values were significantly shorter than during open abdominal surgery. While this may reflect an increased sympathetic outflow in patients undergoing laparoscopic surgery we cannot exclude an additional effect from the increased abdominal and thoracic pressures on PTT. The PTT is a valuable noninvasive monitor, which is easily derived from standard patient monitors and may become a standard additional parameter during anesthesia and surgery just as ST segment monitoring. However, our data (and those of others [8,9]) indicate that PTT is not a reliable marker of beat-to-beat blood pressure. Also the use of PTT as a surrogate marker of pain is still premature, as we cannot exclude cardiovascular influences on the PTT independent of pain and stress (such as occur due to the increase in abdominal pressure). Further studies are needed to assess the interaction between pain and influencing factors (e.g. ventilation mode, vasoactive drugs, blood loss, inspired oxygen concentration, atherosclerosis) on PTT during anesthesia.

\section{ACKNOWLEDGEMENT}

This study was made possible by a grant from the TREND (Trauma RElated Neuronal Dysfunction) consortium (Delft, The Netherlands).

\section{REFERENCES}

[1] Weiss T, Del Bo A, Reichek N, Engelman K. Pulse transit time in the analysis of autonomic nervous system effects on the cardiovascular system. Psychophysiol 1980; 17: 202-7.

[2] Dutch J, Redman S. Psychological stress and arterial pulse transit time. N Z Med J 1983; 96: 266-70.

[3] Smith R, Argod J, Pepin JL, Levy PA. Pulse transit time: an appraisal of potential clinical applications. Thorax 1999; 54: 452-7.

[4] Greenwald S, Olofsen E, Dahan A. Pulse transit time (PTT) reflects changes in anesthetic state during sevolfurane $/ \mathrm{N}_{2} \mathrm{O}$ anesthesia. Anesthesiology 2002; 96: A-544.

[5] Singham S, Voss L, Barnard J, Sleigh J. Nociceptive and anaestheticinduced changes in pulse transit time during general anaesthesia. $\mathrm{Br} \mathrm{J}$ Anaesth 2003; 91: 662-6.

[6] Sharwood-Smith G, Bruce J, Drummond G. Assessment of pulse transit time to indicate cardiovascular changes during obstetric spinal anaesthesia. Br J Anaesth 2006; 96: 100-5.

[7] Ochiai R, Takeda J, Hosako, Sugo Y, Tanaka R, Soma T. The relationship between modified pulse wave transit and cardiovascular changes in isoflurane-anesthetized dogs. J Clin Monit Comput 1999; 15: 493-501.

[8] Payne RA, Symeonides CN, Webb DJ, Maxwell SRJ. Pulse transit time measured from the ECG: an unreliable marker of beat-to-beat blood pressure. J Appl Physiol 2006; 100: 136-41.

[9] Young CC, Mark JB, White W, DeBree A, vender JS, Fleming A. Clinical evaluation of continuous non-invasive blood pressure monitoring: accuracy and tracking capabilities. J Clin Monit 1995; 11: 1659.

[10] Ekstein P, Szold A, Sagie B, Werbin N, Klausner JM, Weinbroum AA. Laparoscopic surgery may be associated with severe pain and high analgesia requirements in the immediate postoperative period. Ann Surg 2006; 243: 41-6.

[11] Ortega AE, Peters JH, Incarbone $\mathrm{R}$, et al. A prospective randomized comparison of the metabolic and stress hormonal responses of laparoscopic and open cholecystectomy. J Am Coll Surg 1996; 183: 249-56.

[12] Cullen DJ, Eger EI. Cardiovascular effects of carbon dioxide in man. Anesthesiology 1974; 41:345-9.

[13] Schächinger H, Weinbacher M, Kiss A, Ritz R, Langewitz W. Cardiovascular indices of peripheral and central sympathetic activation. Psychosom Med 2001; 63: 788-96.

[14] Brundin T, Hedenstriena G, McCarthy G. Effect of intermittent positive pressure ventilation on cardiac systolic time intervals. Acta Anaesthesiol Scand 1976; 20: 278-84.

[15] Fujise K, Matsumoto S, Inada T, Yamada K, Shingu M, Iwasaka T. Influence of age on cardiac pump function during laparoscopic cholecystectomy: measurements by ear densitography. Masui 1994; 43: 1509-14.

[16] Sarton E, Olofsen E, Romberg R, et al. Sex differences in morphine analgesia: An experimental study in healthy volunteers. Anesthesiology 2000; 93 : 1245-54. 